\title{
im4Things: An Ontology-based Natural Language Interface for controlling devices in the Internet of Things
}

\author{
José Ángel Noguera-Arnaldos ${ }^{1}$, Mario Andrés Paredes-Valverde ${ }^{2}$, María del \\ Pilar Salas-Zárate ${ }^{2}$, Miguel Ángel Rodríguez-García ${ }^{3}$, Rafael Valencia- \\ García $^{2}$, José Luis Ochoa ${ }^{4}$

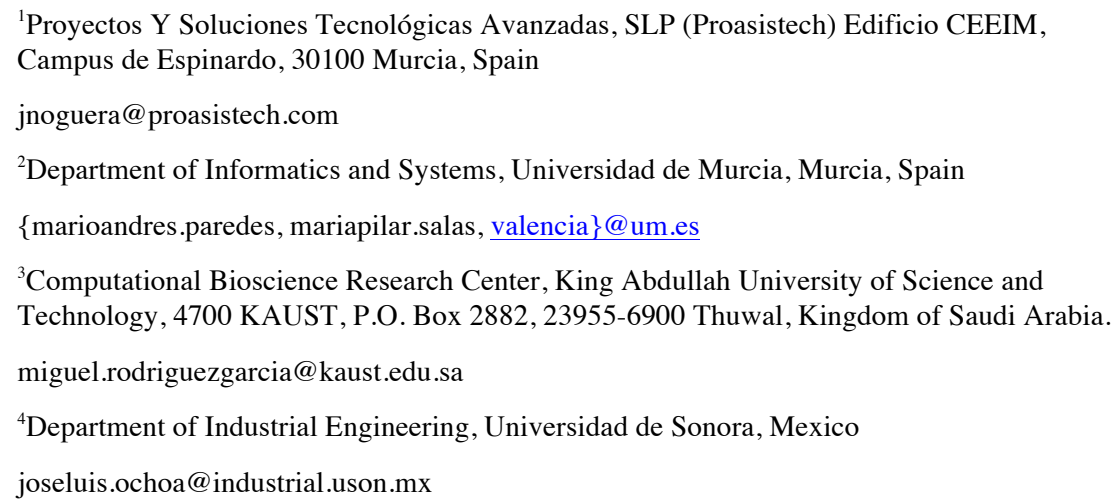

\begin{abstract}
The Internet of Things (IoT) offers opportunities for new applications and services that enable users to access and control their working and home environment from local and remote locations, aiming to perform daily life activities in an easy way. However, the IoT also introduces new challenges, some of which arise from the large range of devices currently available and the heterogeneous interfaces provided for their control. The control and management of this variety of devices and interfaces represent a new challenge for non-expert users, instead of making their life easier. Based on this understanding, in this work we present a natural language interface for the IoT, which takes advantage of Semantic Web technologies to allow non-expert users to control their home environment through an instant messaging application in an easy and intuitive way. We conducted several experiments with a group of end users aiming to evaluate the effectiveness of our approach to control home appliances by means of natural language instructions. The evaluation results proved that without the need for technicalities, the user was able to control the home appliances in an efficient way.
\end{abstract}




\section{Introduction}

The Internet of Things (IoT) refers to the pervasive presence around us of a variety of things or objects such as Radio-Frequency Identification (RFID), sensors, actuators, mobile phones, which, through unique addressing schemes, are able to interact with each other and cooperate with their neighboring smart components to reach common goals[1]. The IoT has been applied in several areas such as transportation systems[2], infrastructure construction, public security, environment protection, intelligent industry, urban management, among others[3]. One of the most attractive markets for the IoT application is the home automation, which refers to the application of computer and information technology for control of home appliances and domestic features [4].

Nowadays, the interaction with home appliances is mainly done through user interfaces based on mechanical buttons, knobs, and more recently, touch sense based buttons. These interfaces only allow users to turn on, turn off, or programming the corresponding device, thus limiting the functionalities that a device can provide, e.g., warnings, fault indication, the cooperation among them, and even, the actuation from remote locations. In this sense, the IoT offers the potential of endless opportunities for new applications and services in the home context that enable users to access and control the home environment, from local and remote locations, in order to carry out daily life activities in an easy way. All that previously mentioned improves the quality of life of the user while at the same time enabling energy efficiency. Despite these advantages, the large range of IoT devices and smart appliances often results to complex systems-of-systems interactions[5], i.e., the wide set of home appliances with diverse requirements and interaction patterns makes the smart home a challenging environment for the design and implementation of intuitive and easy-to-use applications for the endusers. Taking into account that the smart home control system plays a key role on the improvement of the system service quality and the interaction between smart home system and the users[6], it is imperative to provide users with a system that allows them to monitor and control the home environment, from local and remote locations in an easy and intuitive way. Regarding this fact, in this work we present an IoT-based system, known as im4Things, that enables users to control home appliances of different makes, models, and manufacturers, from local and remote locations by means of instant messages based on natural language. The im4Things system combines current technologies such as mobile applications, Natural Language Processing (NLP), Cloud computing, Semantic Web, and Raspberry Pi, an open-source platform used for building electronics projects.

Taking into account that a smart home control system should be affordable, scalable so that new devices can be easily integrated into the system, and it should be user-friendly[7], the im4Things system deals with three main issues. First, the need for an intuitive mechanism for home appliances control. In this sense, the im4Things system provides an instant messaging mobile application which allows 
users to control the home appliances through natural language (NL) messages. The NL paradigm is generally deemed to be the most intuitive from a usage point of view[8]. Also, the im4Things system implements a conversational agent in order to provide to users, in an enjoyable way, information concerning home appliances such as warnings, fault indication, as well as the different states that a home appliance can have during the execution of a user's instruction. Second, the representation and storing of the exchanged information in the context of the Internet of things. This fact is one of the most challenging issues on the IoT context[3]. In this sense, im4Things adopts semantic technologies, particularly ontologies, which represent appropriate modeling solutions for things description, reasoning over data generated by IoT, and communication infrastructure. An ontology is a formal and explicit specification of a shared conceptualization[9]. The ontologies have been successfully applied in domains such as cloud services[10], recommender systems[11], innovation management[12], and sentiment analysis[13], to mention but a few. Third, the need for smart things with proactive behavior, context awareness, and collaborative communications capabilities. In this regard, the im4Thing system provides a low-cost smart item, called im4Things bot, equipped with wireless communication, sensors, memory and elaboration capabilities. Thanks to these features, the im4Things bot not only receives data from home appliances and commands from application platform but also transmits data to the application platform, thus becoming in a generator and receiver of information.

The remainder of the paper is structured as follows. Section 2 presents a review of the literature about home automation and the IoT. The architecture design of the proposed approach, components and interrelationships are described in section 3 . Section 4 presents the evaluation results concerning the effectiveness of the present approach to control home appliances by means of written natural language instructions. Finally, conclusions and future work are presented.

\section{Related works}

Recently, new approaches for home appliances control have been proposed, many of them targeted at the idea of providing speech-controlled home automation. The DIRHA (Distant-speech Interaction for Robust Home Applications) [14] is a system based on the detection and recognition of spoken commands preceded by a small key-phrase, which consist of one up to three words. The current prototype works with a single grammar that recognizes one key-phrase among possible garbage segments. DIRHA recognizes a set of 99 commands of various lengths and, in this regard, a finite-state-grammar was built. $\operatorname{In}[15]$ the authors presented G.H.O.S.T., a voice control system for smart buildings. This system was implemented with the KNX technology, a standardized network communications protocol for intelligent buildings. In G.H.O.S.T., the 
control of the building is performed from a PC via a KNX/IP router and a KNX communication Fieldbus. Also, this interaction is carried out through predefined voice commands, which are focused on the control of lighting, sun-blinds, and air conditioning. On the other hand, Mayordomo[16] is a multimodal (oral, written and a GUI interface) dialogue system focused on the interaction with an Ambient Intelligence environment implemented in a home. Mayordomo implements an ASR (Automatic Speech Recognition) engine as well as a frames-based[17] understanding component. In order to generate answers to the users, Mayordomo makes use of a set of patterns containing information such as home appliance, room, status, among others.

There are some approaches that allow home appliances controlling via the Internet, either e-mail or a Web application. In [4] the authors presented a Raspberry Pi-based home automation system. This system allows the home appliances control through e-mail whose subject represents the instruction to be executed by the Raspberry Pi. The subject must consist of the word ON or OFF followed by a number, which indicates the home appliance to turn on or turn off. Once the corresponding command is executed, the system notifies the user the status of the work done. On the other hand, in[6] the authors presented a smart control system based on the Internet of Things. The system is based on the CoAP [18] protocol, which enables very simple electronics devices the communication over the Internet. This system enables the user to query and control several devices through the Web. For this, the system provides a Web interface with a set of predefined functions, each of which is triggered through a button element.

On the other hand, there are systems focused on home appliances control by means of mobile applications. For instance, in[19] the authors presented a BLEbased (Bluetooth Low Energy)[20] appliance control system. This system implements a "Middle-Device" for the communication between the user, through a mobile application, and home appliances using Arduino, an open-source platform used for building electronics projects. The home appliances control is performed through the selection of predefined functions available on the mobile application. Another example of this kind of systems is presented in[21]. In this work, the authors implemented an Arduino Ethernet based micro-web server for accessing and controlling appliances remotely from an Android application. The mobile application provides a GUI with a set of button elements each of which triggers a specific home appliance action, such as turn on the emergency light, to active the house alarm, and to lock the main door, among others. On the other hand, in[22] the authors presented a smart switch control system. This system provides a GUI implemented using Web technologies but packed as native applications. Also, it implements a SOC (System on a Chip) power metering chip through which the switching operation over saving lamps, air conditioners, refrigerators, and microwaves is performed.

Despite that the above-presented works have made a significant contribution to the smart home field, they present certain disadvantages that are addressed by our 
approach. First, works such as[19] implements Bluetooth technology. Despite Bluetooth capabilities are good and most of current smartphones and tablets have integrated this technology, wherewith the cost of the system reduces significantly, it limits the home appliance control within the Bluetooth range of the environment. In the same context, some works such as Mayordomo, DIRHA, and G.H.O.S.T. represent smart home systems which are intended to provide the control of the home environment just from inside the home. On the basis of this scenarios, the present work provides users the advantage of controlling the home environment not just from inside the home but also from remote locations thanks to a Cloud service implemented by the im4Things system. This feature is interesting for busy families or individuals for whom to take the control of the home environment from remote locations can represent time-saving, thus improving their quality of life. Second, some approaches such as Mayordomo, [6], [19], [21], and[22] provide a GUI which must be modified when a new device is added to the smart home system. This fact can confuse to the users due to the continuous changes on the user interface. Also, these changes increase the development effort. In order to address the issue above, the im4Things system provides a mobile application that points to both configure a new device via wireless connection (Bluetooth) and, once the new device is configured, establish communication with the device by means of instant messages based on natural language. In this sense, the user does not perceive any changes on the user interface, due to the new device is added as a new contact, as it occurs in current instant messaging applications such as WhatsApp and Telegram. Third, the user interaction in systems such as DIRHA, G.H.O.S.T., and[4] is performed through the recognition of small key phrases, predefined voice commands, and through an e-mail message whose subject contains the keyword ON/OFF followed by a number, respectively. This fact can be tedious and complicated for end-users due to the need to learn a set of phrases or keywords. In this sense, the present approach provides an instant messaging application through which the users can interact with the home environment through natural language, thus avoiding the need to learn new commands. Also, as was previously mentioned, the natural language paradigm is generally deemed to be the most intuitive from a usage point of view. Besides, the im4Things system provides a conversational agent aiming to interact with the user through natural language, thus enhancing the user experience. Finally, it should be mentioned that the implemented conversational agent exploits the use of ontologies in order to address one of the main limits of this technology, the rigidness of the dialogue mechanism. All above-mentioned features are described in detail in next section. 


\section{3. im4Things system}

According to [23], a generic NLI (Natural Language Interface) architecture requires four main components: (1) input devices that include user interfaces as input data mechanism, (2) a natural language understanding component that analyses the user input and, optionally interacts with domain-specific information, (3) an interaction manager component that maintains information about ongoing interaction, and (4) a natural language generation component that produces the appropriate responses. On the basis of this understanding, in this work we propose an IoT system, known as im4Things, which addresses all above-mentioned features through the implementation of three main components: a mobile application, a Cloud-based service, and a low-cost smart electronic device. The mobile application serves as the input data mechanism through which users interact with the system in natural language aiming to control the home environment. The Cloud-based service serves two functions: managing the interaction between the users and the home environment, and the natural language understanding. The first function is performed by means of the establishment of a bi-directional communication between the entities involved. This communication is based on the Extensible Messaging and Presence Protocol (XMPP)[24], an open and extensible XML-based protocol. On the other hand, the Natural Language understanding function aims to process the users' messages in order to detect the home appliance and the action it must execute. This processing is based on an ontology that describes the home appliances domain. Finally, the smart electronic device, known as im4Things bot, serves not only as a bridge between the application platform and the home environment but also as a natural language generator that provides information about the status of a home appliances during the execution of a task as well as the home environment status. Concerning this natural language generation process, the im4Things bot implements an AIMLbased (Artificial Intelligence Markup Language)[25] dialog system.

In a nutshell, the im4Things system works as follows. First, the user adds and configures a new home appliance through the mobile application. Actually, the configuration process occurs between the mobile application and the im4Things bot that control the corresponding home appliance. This configuration is performed by means of Bluetooth connection. Once the new device is configured, the user, which automatically becomes the administrator of the device, is able to interact with it through natural language messages. Also, the administrator is able to provide access to the device to other users who, in addition to controlling the home appliance, also will be informed about any change on the home appliance's status. Then, when a user needs performing a housework by means of a specific device, the user sends a message to the corresponding im4Things bot. Before the message is received by the target device, it is processed by the im4Things Cloud service through the understanding module. This process aims to obtain all semantic information from the words contained in the message and determine the 
command that must be executed by the target device. As previously mentioned, this natural language processing is based on a home appliances ontology, which describes, among other things, the states, sensors, actions, alerts and services that a home appliance provides. When the im4Things bot receives the message, it interacts with the home appliance in order to execute the corresponding action. Finally, once the home appliance executes the command, the im4Things bot notifies the user, on natural language, about the result (success or failure) of the command execution.

Besides the above-mentioned process, the im4Things bot is also able to notify the users about the home appliance's state changes. Therefore, once a home appliance's alert is triggered, the im4Things bot sends to the im4Things Cloud service a natural language expression concerning the situation. Then, the im4Things Cloud service notifies the users subscribed to the home appliance. Finally, it is worth mentioning that im4Things system allows users to control home appliances from the local network and remote locations.

Figure 3.1 shows the architecture proposed in this work which, as previously mentioned, is composed of three main modules, (1) the im4Things App, (2) the im4Things Cloud Service, and (3) the im4Things Bot. These modules are described in detail in the next sections. 


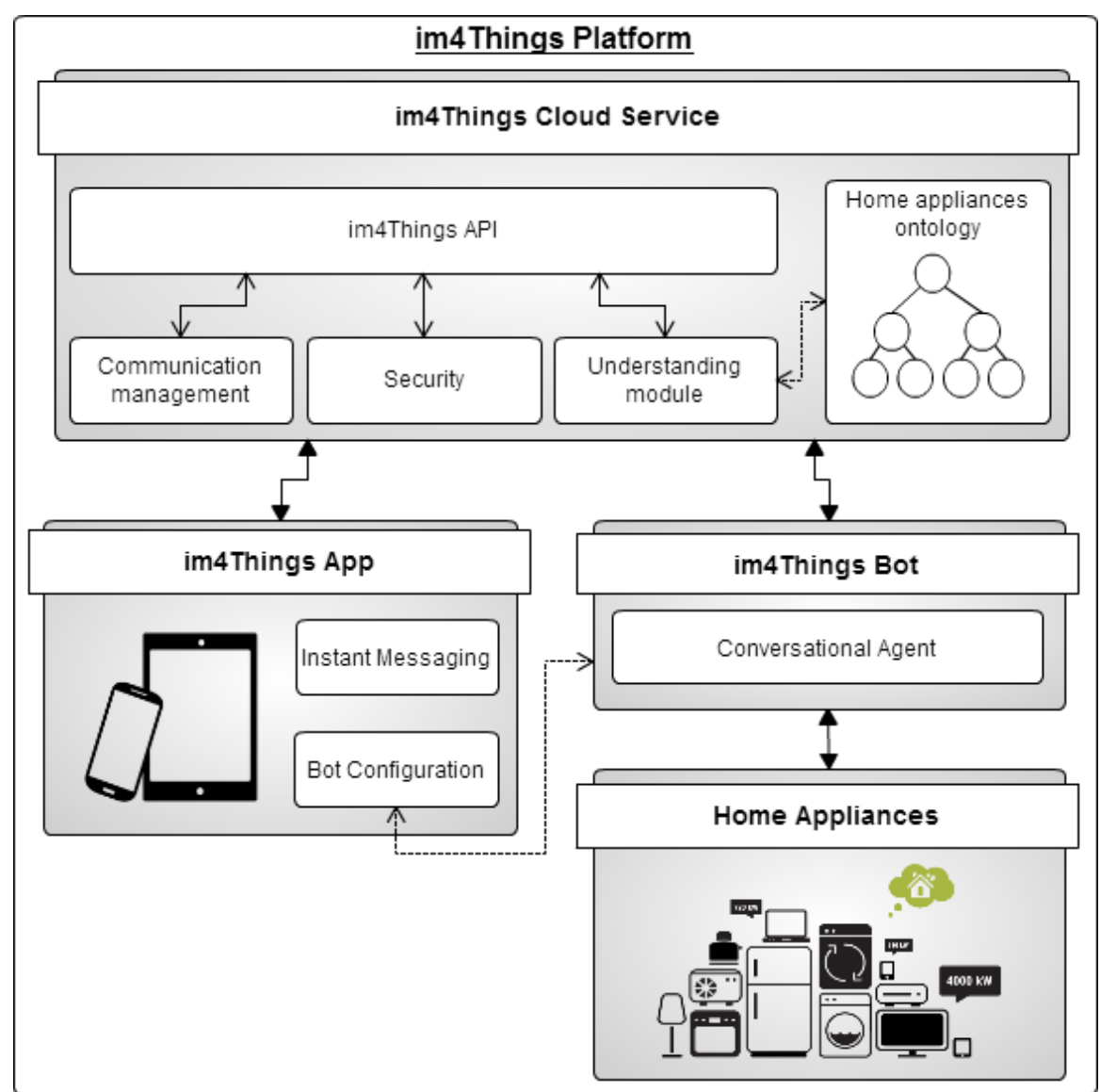

Fig. 3.1im4Thing's architecture.

\section{1 im4Things App}

The im4Things App is a mobile application through which users can establish a communication with different entities, either humans or Bots. The functionalities provided by this application can be grouped into two main categories: (1) Bot Configuration, which are related to the Bot's registration and configuration, and (2) Instant Messaging, which concern to the sending and receiving of messages to and from humans and Bots. In the following sections, the functionalities groups above are described in detail.

\subsubsection{Bot Configuration}


This module allows to register and configure a Bot in order to make it part of the home appliances network, thus allowing its management through natural language instructions. The Bot registration is performed by a user, which automatically becomes the administrator of the Bot. The first step of the registration process is the identification of the Bot through a wireless connection (Bluetooth). Once the Bot is recognized, it sends its unique identifier to the user in order to allow the synchronization between the user and the Bot. The final step consists of creating the Bot profile consisting of a nickname and a picture, which will enable the easy identification of the Bot. Also, this profile includes some information concerning connection properties such as the IP address, which will allow the Bot to communicate with another entity via the Internet. The Bot profile is sent to the im4Things Cloud module in order to allow the remote access to the Bot.

Once the Bot has been registered and configured, its profile can be shared among the members of the home appliances network, in order to establish a communication with it. Also, the Bot's administrator can create instant messaging groups, thus enabling the interaction between the group's members and the corresponding home appliance. This feature is explained in the following section.

\subsubsection{Instant Messaging}

The Instant Messaging module provides a graphical interface where users can write natural language instructions aiming that home appliance performs an activity or informs about its current state. This communication is based on the XMPP protocol, which allows the management of secure instant messaging sessions. Among the functionalities provided by this module are:

- User profile management. This functionality refers to the creation and edition of the user profile which helps to identify a user based on its nickname or avatar.

- Instant messaging. It allows the communication among the entities through instant messages.

- Group chat. This feature allows the administrator to control several home appliances from a unique chat window. Also, by means of this functionality, the administrator can add people to the group, thus allowing them to control all home appliances members of the group.

- User's presence. It refers to the fact that a user has agreed that another entity is allowed to view its presence, thus allowing the communication establishment among them. This feature is especially important in order to allow that a Bot can be visible or hidden to a certain group of users. 


\section{2 im4Things Cloud service}

The im4Thing system has been implemented on a Cloud-based architecture in order to take advantage of Cloud computing technology such as low costs, immediate access to hardware resources, scalability,[26] and, especially, the ability to deliver the im4Things system as a service over the Internet, thus allowing users to control their home appliances from remote locations. The im4Things Cloud service is composed of three main modules: the communication management module, security module, and the understanding module. The interaction between the entities, whether user or im4Things bot, and the im4Things Cloud service is performed through a REST-based API. All the modules above are described in next sections.

\subsection{1 im4Things API}

The im4Things API is a REST-based application programming interface which describes the services provided by the im4Thing Cloud such as the entities' management, authentication, and instant messaging. Through this API, both im4Things App and im4Things bot interact with the im4Things Cloud service.

\subsubsection{Communication management}

The main objective of this module is the communication management among the system's entities. This communication is based on MongooseIM, a customizable and scalable platform for instant messaging for social media, gaming and telecommunications. In this sense, the present module takes advantage of several MongooseIM features such as multi-user chat, Websockets and privacy.

In the im4Things system, the communication can be established in the following different ways: human to human, human to bot, and bot to human. Therefore, one of the most critical points of this module is ensuring the quality and continuity of the service through a correct management of the instant messaging traffic. In this sense, we established a priority queue for the instant messaging management based on the participating entities. The present module distinguishes three priority levels:

- Priority 0 . This level refers to the human-to-human interaction. In this case, the message is transmitted as it is.

- Priority 1 . This level corresponds to the human-to-bot interaction. In this level, the message is interpreted as an instruction to the bot, therefore, it is analyzed in order to detect the target bot as well as the specific command to be executed by the latter. 
- Priority 2. The higher priority level corresponds to the bot-to-human interaction. In this case, the message is interpreted as a bot notification, therefore, it is sent to all users subscribed to the bot. The bot-to-human interaction has the higher priority because the bot can notify the users about warning or alerts that demand the instant or quick participation of the im4Things bot's administrator.

\subsubsection{Security}

The im4Things approach uses a token-based authentication method. In this context, a token contains the security identity of an entity which allows the latter to be identified by the server. Therefore, each request made by an entity must contain the token. Also, in order to ensure the data security, all messages are encrypted using SSL certificates such as 2048 bits and SHA-2.

\subsubsection{Understanding module}

The understanding module is based on a previous work[26] of our research group, which aims to classify a natural language question. The above-mentioned work uses an ontological model for representing all semantic information obtained from the question and for inferring the answer type expected by the user. The present module, for its part, aims to classify the kind of instruction that a user has provided, i.e., this module determines if the instruction is a command that must be executed by the home appliance or just a query of the state of the home appliance. Moreover, this module aims to determine the home appliance that must execute the instruction provided. This is particularly important due to, as was previously mentioned, the instant messaging application allows the creation of chatting groups, where a user can control several devices through a single window. In the chatting groups, it is not necessary to specify the home appliance that must execute the action since the understanding module infers the home appliance based on the semantic information extracted from the message as well as the home appliances ontology.

Aiming to reach the above-mentioned objective, the understanding module performs the natural language processing of the instruction in order to obtain all semantic information from the words contained within it. The most relevant natural language processing techniques implemented by this module are the named entity recognition (NER), lemmatization, and synonym extension. The NER technique seeks to locate and classify words contained in the user's instruction into predefined categories such as home appliances, sensors, alerts, and states, among others. It is worth noting that this technique makes use of the home appliances ontology in order to recognize and classify these entities. With regard 
to the lemmatization and synonym extension techniques, they are used to deal with user's instructions whose elements are not the same contained in the knowledge base (home appliances ontology), e.g. a word could be declared in different verb time, in plural, or it could represent a synonym of some entity contained in the knowledge base. The implementation of these techniques allows reducing cases where the mapping between words contained in the instruction and elements of the knowledge base fails, thus increasing the effectiveness of the system.

Based on the collected information, the system is able to determine the target home appliance, the command it must execute, as well as if the instruction can be executed by the home appliance. On the one hand, the determination of the home appliance and the command becomes a determinant factor in the chatting groups with more than one bot. For instance, let us consider a case where a user is the administrator of two home appliances, a coffee maker and an irrigation system, and the user uses a chatting group to control both appliances. When the user writes the instruction "please make a coffee", the system recognizes, based on the home appliances ontology, a semantic relation between the word "coffee" and the coffee maker. Therefore, the system sends this instruction to the coffee maker instead of the irrigation system. On the other hand, as mentioned above, the system is able to identify if the instruction provided by the user can be executed by the home appliance. For instance, if a user asks the lighting control to make a coffee at the chat window exclusively focused on the control of this appliance, the system will send a message to the user indicating the error. It must be noted that the abovementioned interaction is performed through natural language.

\section{Home appliances ontology}

The home appliances ontology provides a formal representation of the environment in which the im4Things system is placed, i.e. the home appliances domain. In this sense, the home appliances ontology establishes five main classes:

- State. This class represents all possible states that can have a home appliance.

- Sensor. It represents all sensor available in the home appliance.

- Action. This class represents all functionalities provided by the home appliance.

- Alert. It represents the alerts that a home appliance can trigger.

- Service. This class represents all services that a home appliance can perform.

Figure 3.2 shows an excerpt of the home appliances ontology describing the coffee maker context. This ontology excerpt shows the six states that a coffee maker can have. Also, the coffee maker sensors (WaterLevel, Temperature, CoffeeLevel) are shown, these sensors can trigger different alerts (NoCoffee, NoWater, Drink_Ready), for instance, when the water tank is empty, the coffee maker triggers an alert asking the user refilling the tank. It has to be borne in mind 
that this communication is established through natural language messages. This process will be discussed next.

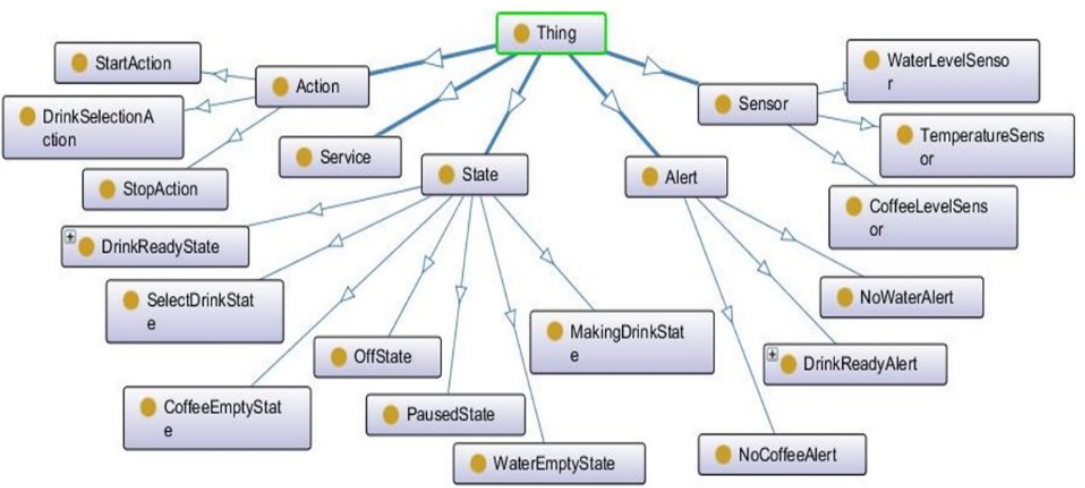

Fig. 3.2An excerpt of the home appliances ontology describing the Coffee maker's context.

\section{3 im4Things Bot}

The im4Things Bot represents the interface between the home appliance and the im4Things top layers. The im4Things Bot has a specific hardware composed of sensors and a control and communication system that interacts with the software in order to execute commands and inform about the home appliance status in an efficient way. This bot is based on the Raspberry Pi hardware, which has been successfully applied in multiple control and voice recognition systems[27]. Also, the im4Thing bot embeds an AIML-based conversational agent which is responsible for simulate a human communication behavior in order to interact with the users through natural language. In the next section, this module is explained in detail.

\subsubsection{Conversational agent}

The conversational agent is based on the dialog system technology. This technology was used to implement a system intended to converse with a human, with a coherent structure. In this sense, the AIML language was used to develop a natural language software agent. The AIML language is able to recognize predefined patterns in a text, and generate answers according to several conditions and states of the system. The AIML language is composed of regular expression patterns associated to a set of answers. These answers are provided to the user when the corresponding pattern is identified. Figure 3.3 shows an excerpt of the definition of a conversational agent. This definition contains two patterns, the first 
one establishes that if the system identifies the phrase "Are you a coffee maker?", it will provide the answer defined by the "template" element. With regard to the second pattern, it is used when the system identifies the phrase "is your name?" preceded by several $(*)$ words.

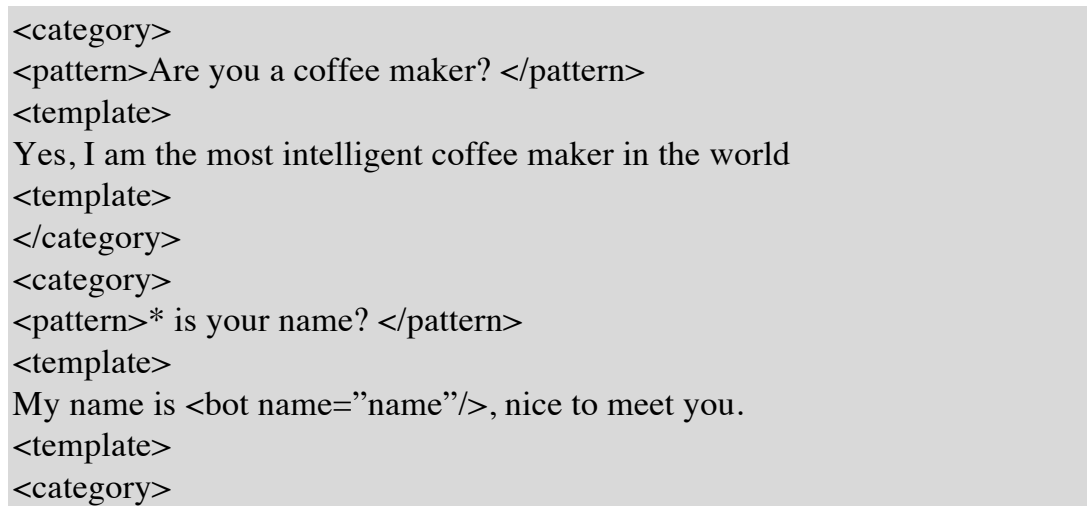

Fig. 3.3Definition of two AIML patterns.

As described above, the AIML language is used to define patterns which produce an answer according to a simple correspondence of words. The rules (pattern matching rules) on which these conversational agents are based to carry out the dialogue are too restrictive and their language understanding capability is very limited[28]. On the basis of this understanding, we have implemented an extended version of the AIML-based structure above shown. The proposed structure takes into account the concepts contained in the home appliances ontology instead of a set of tokens (words). In this sense, the "pattern" element was extended aiming to represent the action, status, and the home appliance identified in the user's instruction. Also, the "action" element was added to the AIML structure; this element indicates the command that the home appliance must execute. Finally, the "template" element includes the answers that the system provides when the command is successfully executed or not. Figure 3.4 shows an excerpt of the Coffee maker's AIML-based patterns. The first pattern represents that the "DrinkSelection" action was found in the user's instruction and that the coffee maker's state is "Pause". Thus, when both conditions are met, the "MAKE_COFFEE" command will be sent to the home appliance. If the command is successfully executed, the system sends to the user the answer contained in the "success" element, otherwise, the system sends the answer contained in the "fail" element. The second pattern refers to a user's question about the state of the home appliance. Finally, it should be remembered that the system is also able to monitor the state of the home appliance and send an alert when there is a problem with it. In this sense, the third pattern refers to the alert indicating that the coffee maker has no water, then the system asks the user refilling the water tank. 


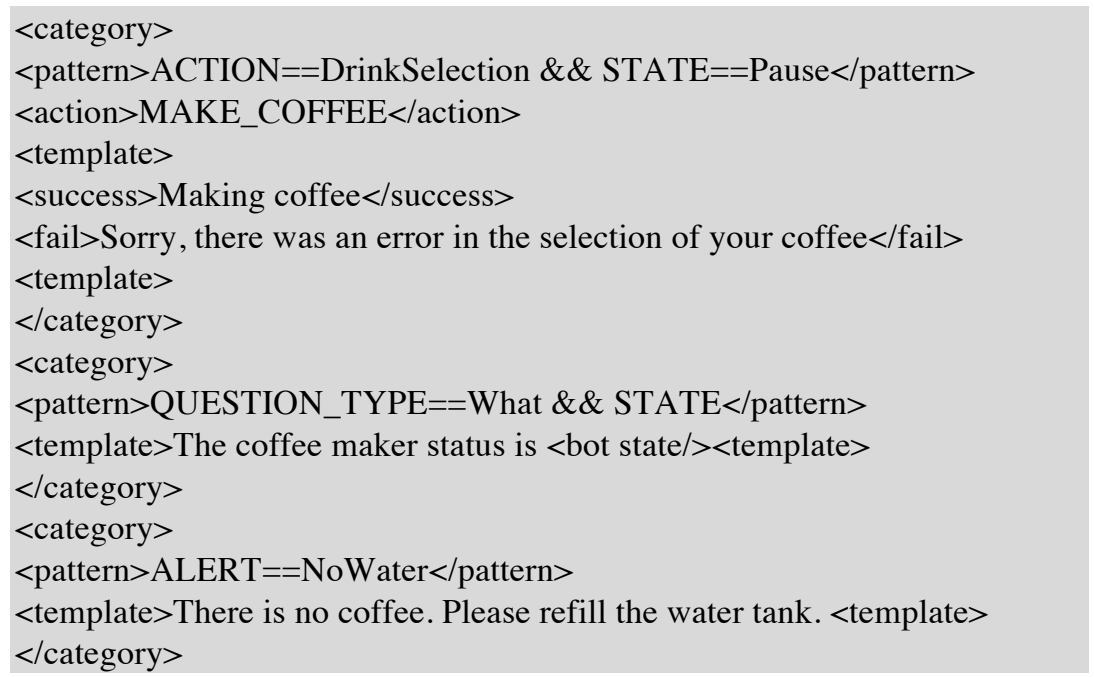

Fig. 3.4An excerpt of the Coffee maker's AIML-base patterns

\section{Evaluation and results}

Despite the fact that the im4Things system is composed by several modules and services, the performed experiments were focused on evaluating the effectiveness of the present approach to control home appliances by means of written natural language instructions. In this sense, we performed a set of experiments with a first functional prototype and a set of real-world end users. This prototype has been configured for the control of five different home appliances: a coffee maker, an irrigation system, an internal lighting control, blinds, and an air heater. The overall evaluation process is described below.

\subsection{Subjects}

In order to evaluate the im4Things prototype with real-world users, the experiments were performed at the premises of the CEEIM (European Centre of Enterprises and Innovation of Murcia) located in the University of Murcia, Spain. The set of participants was composed of 5 people unrelated to the research group of which the authors are members. The age of the participants ranges from 20 to 43 years old. The participants must have two important characteristics: On the one hand, the participants must have knowledge about the use of mobile applications, particularly the use of instant messaging applications. This fact did not constitute a 
problem due to all participants have experience using well-known instant messaging applications such as WhatsApp ${ }^{\circledR}$ and WeChat ${ }^{\circledR}$. On the other hand, the users must have knowledge about the services provided by the home appliances involved in the im4Things prototype. In this sense, the participants were informed of the general purpose of the study and they received an initial description of all services provided by each home appliance as well as of the im4Things mobile application.

\subsection{Procedure}

The first step of the evaluation process consisted of providing the participants a general description of the im4Things mobile application as well as of all features provided by each home appliance configured in the im4Things prototype, i.e., the coffee maker, the irrigation system, the internal lighting control, the blinds, and the air heater. After that, the im4Things mobile application was installed in the smartphones of the participants. Then, one participant was asked to add and configure all above-mentioned home appliances to the im4Things system. It is worth remembering that the im4Things mobile application allows users to interact with only one device through an individual chat window, or with more than one device through the same chat window thanks to the chatting group feature. On the basis of this understanding, the user-bot interaction performed through a chatting group with more than one im4Things bot, is the most difficult scenario from a system point of view due to the fact that, aside from the need of obtaining the command that the home appliance must execute, the system needs to determine the target home appliance. Therefore, the users were asked to create a chatting group in such a way as to enable them to control all home appliances through the same chat window, i.e., there was a total of five chatting groups each of which was integrated by one user and the five home appliances. Once all chatting groups were created, the users were asked to control the home appliances through natural language instructions. It is worth mentioning that the users used the mobile application at the premises of the CEEIM as well as from remote locations. On the other hand, due to the fact that this evaluation involves human participation, we put special attention on checking that the users were aware that these experiments were focused on evaluating the im4Things system and not the users since this fact can influence the test results[29].

Finally, once the im4Things mobile application was used by the participants along fifteen days, we analyzed all messages provided by them and used the precision, recall and F-measure metrics to obtain statistic values that allow us to determine the effectiveness of the present approach to control home appliances by means of written natural language instructions. The evaluation results are shown in the following section. 


\subsection{Results}

As mentioned earlier, the present evaluation used the primary metrics precision and recall, and their harmonic mean, known as the F-measure [30]. These metrics have been commonly applied in information retrieval experiments [31] and natural language interfaces development [8], [26]. These metrics can be specified by using the terminology of false positives and false negatives [32]. In this context, true positives refer to the user's instructions that were successfully executed by the home appliance. The false positives refer to the user's instructions that were executed by the home appliances but they were not what user expected. Furthermore, the false negatives refer to the user's instructions that were not executed by the home appliance, but they should have been executed and the user's instructions that were executed by the home appliances but they were not what user expected. Based on the aforementioned, the precision, recall and Fmeasure formulas are shown in Figure 4.1.

\section{Precision $=$ True Positives $/$ (True Positives + False Positives $)$ \\ Recall $=$ True Positives $/$ (True Positives + False Negatives $)$ \\ F-Measure $=(2 *$ Precision $*$ Recall $) /($ Precision + Recall $)$}

Fig. 4.1Precision, Recall and F-measure formulas.

Table 4.1 shows the results obtained by each home appliance as well as by the whole system. From this table we can appreciate that a total of 745 instructions were provided by the group of participants, being the coffee maker, the home appliance that received the highest number of instructions. Conversely, the blinds obtained the lowest number of instructions. Also, we can see the total number of instructions that were correctly executed by the home appliance (TP - True Positives), the number of instructions that were executed by the home appliances but they were not what user expected (IE - Incorrectly executed), and the number of instructions that were not executed by the home appliance (NE - No executed). These two latter groups make up the False Negatives group.

With regard to the precision, recall and F-measure scores, the coffee maker and the irrigation system obtained the best results. Meanwhile, the blinds obtained the lowest recall score. On the other hand, the air heater obtained the lowest precision score. In general terms, the im4Things system achieved promising results with an average precision of 0.9262 , a recall of 0.895 , and an F-measure of 0.9102 .

\begin{tabular}{llllllll}
\hline Home appliance & Total & TP & IE & NE & Precision & Recall & F-measure \\
\hline Coffee maker & 200 & 185 & 10 & 5 & 0.9487 & 0.925 & 0.9367 \\
Irrigation system & 160 & 148 & 8 & 4 & 0.9487 & 0.925 & 0.9367 \\
Internal lighting control & 185 & 165 & 15 & 5 & 0.9166 & 0.8918 & 0.9041 \\
\hline
\end{tabular}




\begin{tabular}{llllllll}
\hline Blinds & 80 & 68 & 6 & 6 & 0.9189 & 0.85 & 0.8831 \\
Air heater & 120 & 106 & 12 & 2 & 0.8983 & 0.8833 & 0.8907 \\
& 745 & 672 & 51 & 22 & & & \\
Average & & & & & 0.9262 & 0.8950 & 0.9102 \\
\hline
\end{tabular}

Table 4.1 Evaluation results.

\subsection{Discussion}

The results obtained by the evaluation above prove that without the need for technicalities, the user was able to control the home appliances in an efficient way through the im4Things system. However, despite the results obtained seem encouraging, the im4Things system presented specific issues which are described next.

Based on a detailed analysis of the users' instructions, we ascribe the Falsenegatives (incorrectly executed and non-executed instructions) instructions to next reasons: (a) non-identification of keywords that help to determine the home appliance and the action to be executed, (b) user location within the home, and (c) complexity of the users' instructions. With respect to the first reason given above, some users' messages did not contain words describing the home appliance or the action to be executed, but rather they implied it. For instance, the user's message "It's too hot" does not describe the target home appliance or an action to be executed. Instead, it describes the current state of the room where the user is located. An interesting question is what else can be the system from such messages. In this sense, we are convinced that our approach can be extended to provide a more robust behavior with respect to this kind of messages. Also, we believe this improvement might be achieved through the implementation of new methods that allow us to consider a wider semantic context of the users' messages as well as the user location. Concerning user location within the home, we perceived that the main reason why the Internal lighting control got the worst results is because the users' messages were referring to a determined luminaire within the room where the user was located, e.g., "Turn off the left lamp". Based on this understanding, our approach needs to be improved through the integration of more sensors that allow the system to obtain information of the home environment and the user location within the home, thus enabling the system to take decisions based on the data obtained by the sensors. Finally, concerning the complexity of the instructions, we detected that some users' instructions required the coordination and participation of more than one home appliance, as well as of information about the current state of the room where the home appliances are located, e.g., if there are people in the room. An example of this kind of messages is "If there are not people in the main room, turn off the lights and lowers the blinds". As we can see the instruction above demands the coordination of two 
home appliances as well as of information about the main room. Once again, it is present the need for sensors that provide information about the room status as well as mechanisms that allow im4Things bots to cooperate among them in order to perform this kind of complex activities. From this perspective, we conclude that the im4Things system was slightly limited in dealing with complex instructions. However, we are convinced that our approach can be improved to address all above- mentioned issues without sacrificing or weakening the user expressiveness. All that previously mentioned can be achieved through the exploitation of the protocols and technologies involved in the present work. In this sense, the next section presents a detailed description of the future research.

\section{Conclusions and future research}

In this work, we presented a Cloud-based system to monitoring and controlling the home environment from local and remote locations. This system allows users to perform the aforementioned activities through natural language instructions by using an instant-messaging application. The natural language paradigm adopted is characterized by a flexible and intuitive way of interaction from a usage point of view. Furthermore, the user interaction paradigm adopted in this work was enhanced through the implementation of a conversational agent aiming to interact with the user through natural language, thus enhancing the user experience.

The im4Things system took advantage of technologies such as Semantic Web and the Raspberry Pi. On the one hand, the Semantic Web enabled the definition of a home appliances ontology upon which the system was able to infer the device and instruction to be executed from the natural language messages. This ontologybased approach proved to be an efficient way to process the natural language users' messages. However, the ontology needs to be extended aiming to take into account a wider set of devices available on the home environment as well as the user location. On the other hand, the Raspberry Pi technology has proved to be a smart, economic an efficient technology for implementing home appliances control through the Internet. However, it is still having small storage space and low computational efficiency. In this sense, we are convinced that this technology will be enhanced in such a way as the im4Things bot here proposed will be able to perform more demanding processes thus allowing it to become a smarter device able to cooperate with other devices to perform complex activities.

Furthermore, in this paper we presented a set of experiments performed in order to evaluate the effectiveness of the im4Things system to control home appliances by means of natural language instructions. The results obtained seem encouraging and make us believe that the system represents a solid base to face the IoT application in the home environment. However, we are aware that the system here proposed may be further expanded and improved with capabilities such as the integration of more sensors and actuators that allow to obtain 
information of the home environment aiming to provide suggestions to the user about energy efficiency and even allow the system itself takes certain decisions [33]. This improvement would require the extension of the home appliances ontology proposed in this work as well as of the functionalities provided by the im4Things bot. Regarding this latter issue, another potential future development refers to the communication between the im4Things bots. This feature will enable a im4Things bot to be informed about the status and configuration of other devices, thus allowing it to perform activities that involve the participation of more than one device, such as "turn off all lights that are in the same room with blinds up". It is worth noting that the correct execution of this request will provide users a complete impression of a smart home.

Finally, concerning natural language interaction, we are considering the use of emoticons in the instant messaging application. This feature will enable users to express their needs through a set of symbols in an easier and more simple way. Despite the fact that this feature might represent an outstanding advantage, its correct implementation in the present context will demand the development of better natural language processing mechanisms.

Acknowledgments María del Pilar Salas-Zárate and Mario Andrés Paredes-Valverde are supported by the National Council of Science and Technology (CONACyT) and the Mexican government.

\section{References}

[1] D. Giusto, A. Iera, G. Morabito, and L. Atzori, Eds., The Internet of Things. New York, NY: Springer New York, 2010.

[2] C.-R. Dow, D.-B. Nguyen, S.-C. Wang, S.-F. Hwang, M. F. Tsai, C.-R. Dow, D.-B. Nguyen, S.-C. Wang, S.-F. Hwang, and M. F. Tsai, "A Geo-Aware Taxi Carrying Management System by Using Location Based Services and Zone Queuing Techniques on Internet of Things, A Geo-Aware Taxi Carrying Management System by Using Location Based Services and Zone Queuing Techniques on Internet of Things," Mob. Inf. Syst. Mob. Inf. Syst., vol. 2016, 2016, p. e9817374, Jan. 2016.

[3] L. Atzori, A. Iera, and G. Morabito, "The Internet of Things: A survey," Comput. Netw., vol. 54 , no. 15 , pp. $2787-2805$, Oct. 2010.

[4] S. Jain, A. Vaibhav, and L. Goyal, "Raspberry Pi based interactive home automation system through E-mail," in 2014 International Conference on Optimization, Reliabilty, and Information Technology (ICROIT), 2014, pp. 277-280.

[5] I. Chatzigiannakis, J. P. Drude, H. Hasemann, and A. Kröller, "Developing Smart Homes Using the Internet of Things: How to demonstrate Your System," in Distributed, Ambient, and Pervasive Interactions, N. Streitz and P. Markopoulos, Eds. Springer International Publishing, 2014, pp. 415-426.

[6] J. Zhu, X. Jia, and X. Q. Mei, "Smart Home Control System Based on Internet of Things," Appl. Mech. Mater., vol. 738-739, pp. 233-237, Mar. 2015.

[7] R. Piyare and M. Tazil, "Bluetooth based home automation system using cell phone," in 2011 IEEE 15th International Symposium on Consumer Electronics (ISCE), 2011, pp. 192-195. 
[8] P. Cimiano, P. Haase, J. Heizmann, M. Mantel, and R. Studer, "Towards portable natural language interfaces to knowledge bases - The case of the ORAKEL system," Data Knowl. Eng., vol. 65, no. 2, pp. 325-354, May 2008.

[9] R. Studer, V. R. Benjamins, and D. Fensel, "Knowledge engineering: Principles and methods," Data Knowl. Eng., vol. 25, no. 1-2, pp. 161-197, Mar. 1998.

[10] M. Á. Rodríguez-García, R. Valencia-García, F. García-Sánchez, and J. J. Samper-Zapater, "Ontology-based annotation and retrieval of services in the cloud," Knowl.-Based Syst., vol. 56, pp. 15-25, Jan. 2014.

[11] L. O. Colombo-Mendoza, R. Valencia-García, A. Rodríguez-González, G. Alor-Hernández, and J. J. Samper-Zapater, "RecomMetz: A context-aware knowledge-based mobile recommender system for movie showtimes," Expert Syst. Appl., vol. 42, no. 3, pp. 12021222, Feb. 2015

[12] R. García, M. Ángel, R. Valencia García, G. Alcaraz Mármol, and C. Carralero, “Open Idea: plataforma inteligente para gestión de ideas innovadoras," Open Idea: an intelligent platform for managing innovative ideas, Sep. 2014.

[13] I. Peñalver-Martinez, F. Garcia-Sanchez, R. Valencia-Garcia, M. Á. Rodríguez-García, V. Moreno, A. Fraga, and J. L. Sánchez-Cervantes, "Feature-based opinion mining through ontologies,” Expert Syst. Appl., vol. 41, no. 13, pp. 5995-6008, Oct. 2014.

[14] A. Katsamanis, I. Rodomagoulakis, G. Potamianos, P. Maragos, and A. Tsiami, "Robust far-field spoken command recognition for home automation combining adaptation and multichannel processing," in 2014 IEEE International Conference on Acoustics, Speech and Signal Processing (ICASSP), 2014, pp. 5547-5551.

[15] J. Vanus, M. Smolon, J. Koziorek, and R. Martinek, "Voice Control of Technical Functions in Smart Home with KNX Technology," in Computer Science and its Applications, J. J. (Jong H. Park, I. Stojmenovic, H. Y. Jeong, and G. Yi, Eds. Springer Berlin Heidelberg, 2015, pp. 455-462.

[16] G. Espejo Pérez, N. Ábalos Serrano, R. López-Cózar Delgado, Z. Callejas Carrión, and D. Griol Barres, "Sistema Mayordomo: uso de un entorno de inteligencia ambiental a través de un sistema de diálogo multimodal," Mayordomo system: using an ambient intelligence environment through a multimodal dialogue system, Oct. 2010.

[17] J. Allen, Natural Language Understanding, 2 edition. Redwood City, Calif: Pearson, 1994.

[18] Z. Shelby, K. Hartke, and C. Bormann, "The Constrained Application Protocol (CoAP)." [Online]. Available: https://tools.ietf.org/html/rfc7252. [Accessed: 26-Apr-2016].

[19] H. Matsuoka, J. Wang, L. Jing, Y. Zhou, Y. Wu, and Z. Cheng, "Development of a control system for home appliances based on BLE technique," in 2014 IEEE International Symposium on Independent Computing (ISIC), 2014, pp. 1-5.

[20] C. Gomez, J. Oller, and J. Paradells, "Overview and Evaluation of Bluetooth Low Energy: An Emerging Low-Power Wireless Technology,” Sensors, vol. 12, no. 9, pp. 11734-11753, Aug. 2012.

[21] S. Kumar, "Ubiquitous Smart Home System Using Android Application,” Int. J. Comput. Netw. Commun., vol. 6, no. 1, pp. 33-43, Jan. 2014.

[22] G. Jie, C. Bo, Z. Shuai, C. Junliang, G. Jie, C. Bo, Z. Shuai, and C. Junliang, "CrossPlatform Android/iOS-Based Smart Switch Control Middleware in a Digital Home, CrossPlatform Android/iOS-Based Smart Switch Control Middleware in a Digital Home," Mob. Inf. Syst. Mob. Inf. Syst., vol. 2015, 2015, p. e627859, Jul. 2015.

[23] R. W. Smith, "Natural Language Interfaces," in Encyclopedia of Language \& Linguistics, $2^{\circ}$ ed., Oxford: Elsevier, 2006, pp. 496-503.

[24] P. S.-A. Tronçon Kevin Smith, Remko, XMPP: The Definitive Guide. .

[25] "Alicebot and AIML Documentation (A.L.I.C.E. AI Foundation)." [Online]. Available: http://www .alicebot.org/documentation/. [Accessed: 25-Apr-2016].

[26] S. Marston, Z. Li, S. Bandyopadhyay, J. Zhang, and A. Ghalsasi, "Cloud computing - The business perspective,” Decis. Support Syst., vol. 51, no. 1, pp. 176-189, Apr. 2011. 
[27] L. F. D. Haro, R. Cordoba, J. I. Rojo Rivero, J. Diez de la Fuente, D. Avendano Peces, and J. M. Bermudo Mera, "Low-Cost Speaker and Language Recognition Systems Running on a Raspberry Pi," Lat. Am. Trans. IEEE Rev. IEEE Am. Lat., vol. 12, no. 4, pp. 755-763, 2014.

[28] A. Augello, G. Pilato, G. Vassallo, and S. Gaglio, "Chatbots as Interface to Ontologies," in Advances onto the Internet of Things, S. Gaglio and G. L. Re, Eds. Springer International Publishing, 2014, pp. 285-299.

[29] E. Kaufmann and A. Bernstein, "Evaluating the usability of natural language query languages and interfaces to Semantic Web knowledge bases," Web Semant. Sci. Serv. Agents World Wide Web, vol. 8, no. 4, pp. 377-393, Nov. 2010.

[30] Y. Yang and X. Liu, "A Re-examination of Text Categorization Methods," in Proceedings of the 22Nd Annual International ACM SIGIR Conference on Research and Development in Information Retrieval, New York, NY, USA, 1999, pp. 42-49.

[31] G. Hripcsak and A. S. Rothschild, "Agreement, the F-Measure, and Reliability in Information Retrieval," J. Am. Med. Inform. Assoc. JAMIA, vol. 12, no. 3, pp. 296-298, 2005.

[32] Advanced Data Mining Techniques. Berlin, Heidelberg: Springer Berlin Heidelberg, 2008.

[33] G. Pilato, A. Augello, and S. Gaglio, "Modular knowledge representation in advisor agents for situation awareness," Int. J. Semantic Comput., vol. 05, no. 01, pp. 33-53, Mar. 2011. 